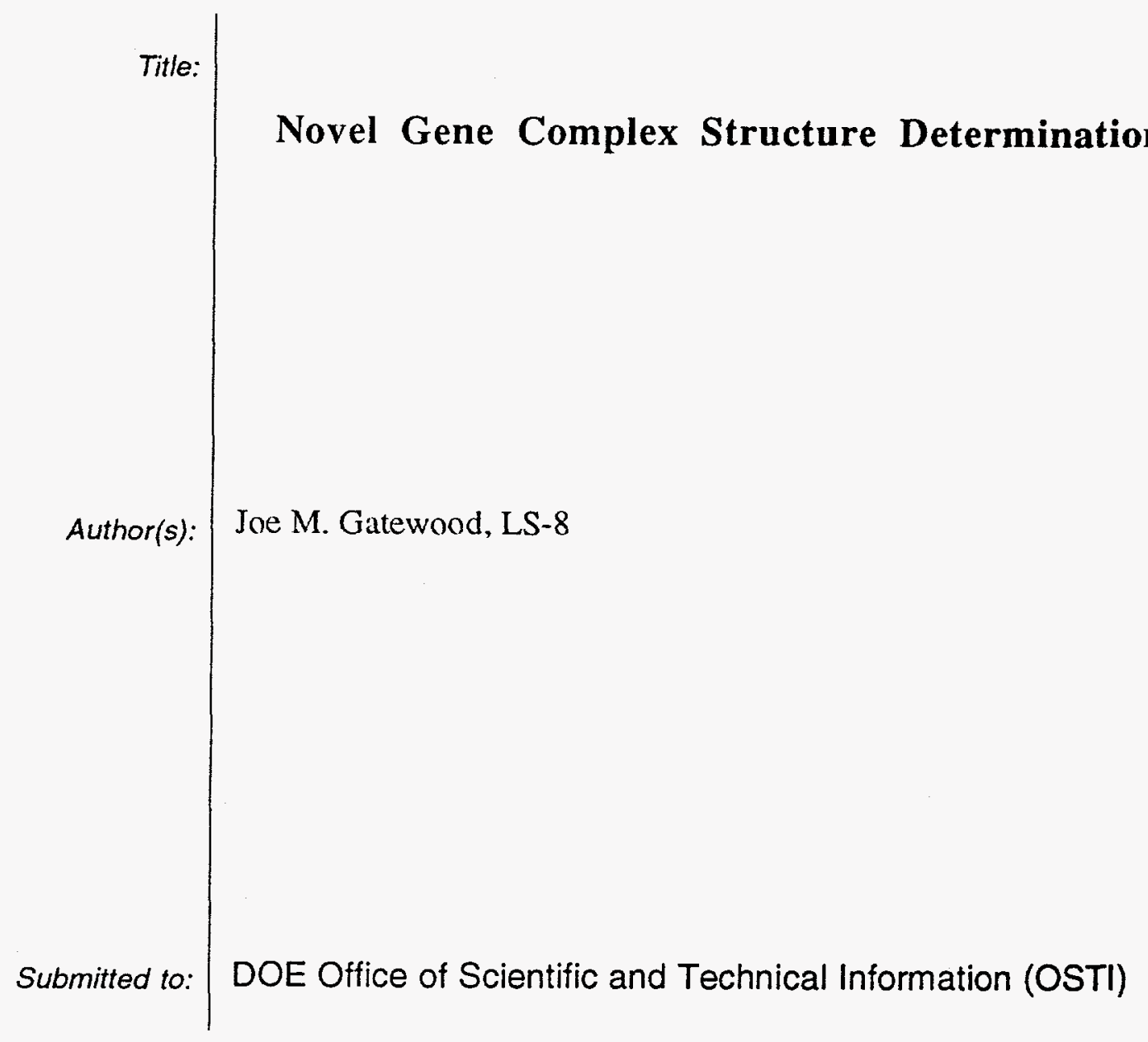

DISCLAIMER

This report was prepared as an account of work sponsored by an agency of the United States Government. Neither the United States Government nor any agency thereof, nor any of their employees, makes any warranty, express or implied, or assumes any legal liability or responsibility for the accuracy, completeness, or usefulness of any information, apparatus, product, or process disclosed, or represents that its use would not infringe privately owned rights. Reference herein to any specific commercial product, process, or service by trade name, trademark, manufacturer, or otherwise does not necessarily constitute or imply its endorsement, recommendation, or favoring by the United States Government or any agency thereof. The views and opinions of authors expressed herein do not necessarily state or reflect those of the United States Government or any agency thereor.

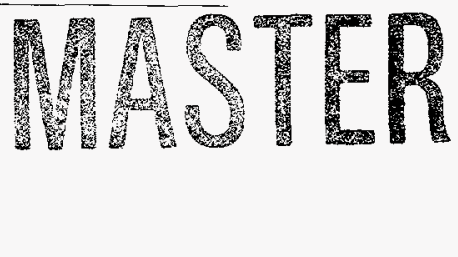

Los Alamos National Laboratory, an affirmative action/equal opportunity employer, is operated by the University of Calitomia for the U.S. Department of Energy under contract W-7405-ENG-36. By acceptance of this article, the publisher recognizes that the U.S. Government retains a nonexclusive, royaltyfree license to publish or reproduce the published form of this contribution, or to allow others to do so, for U.S. Government purposes. Los Alamos National Laboratory requests that the publisher identify this article as work pertormed under the auspices of the U.S. Department of Energy. Los Alamos National Laboratory strongly supports academic freedom and a researcher's right to publish; as an institution, however, the Laboratory does not endorse the viewpoint of a publication or guarantee its technical correctness. 


\section{DISCLAMIER}

Portions of this document may be illegible in electronic image products. Images are produced from the best available original document. 


\title{
Novel Gene Complex Structure Determination
}

\author{
Joe M. Gatewood*
}

\begin{abstract}
This is the final report of a one-year, Laboratory-Directed Research and Development (LDRD) project at the Los Alamos National Laboratory (LANL). "Operative" chromatin containing exclusively the minor histone variants was successfully isolated. Linker histone $\mathrm{H} 1$ is quantitatively missing from operative chromatin. One of the aims of this proposal was to determine the proteins responsible for stabilizing operative chromatin. This chromatin is stabilized by microtubule proteins tau and tubulin. Another goal of this project was the structural characterization of operative chromatin nucleosomes. Using solution scattering, nucleosomes containing the minor variants were shown to be structurally distinct from major variant containing nucleosomes. The unusual structure and stabilization of operative chromatin by microtubule proteins provides a possible mechanism for direct interaction of transcription machinery with specific chromatin domains.
\end{abstract}

\section{Background and Research Objectives}

The major macromolecular complexes that support human life and reproduction have megadalton molecular weights. Due to the large size and complexity of these assemblies, structural investigations require high resolution characterization of the smaller subunits combined with low resolution, higher order information. Multidimensional NMR and $\mathrm{X}$-ray crystallography provide high resolution information while techniques such as scanning force and electron microscopy provide low resolution higher order information. A particularly powerful technique for complementing NMR and X-ray crystallography is small angle solution scattering. LANL expertise and instrumentation provide one of the rare sites in the country where this type of higher order information can be obtained.

Small angle neutron and $x$-ray scattering does not require crystals or sample fixation and the sample can be maintained under a wide range of chemical and physical conditions. As a result, the macromolecules can be examined in as close to a native state as possible. These advantages are opposed by the fact that a precise three-dimensional picture of the

\footnotetext{
*Principal Investigator, e-mail: gatewood@lanl.gov
} 
molecule cannot be obtained by small-angle solution scattering alone. However, the data from solution scattering contains precise information about the overall size and shape of the molecule which places constraints upon the possible structure(s). This information, in combination with other structural data and model fitting, can give a precise picture of the molecule as well as the dynamics of any flexible regions.

$\mathrm{X}$-ray and neutron scattering are, in principle, quite similar but there are important differences that make them complementary techniques in practice. First and foremost, neutrons are scattered by atomic nuclei and this allows the effective use of the technique of contrast variation. When the scattering length density of a molecule or portions of a molecule are matched by the solvent, the "contrast matched" elements are indistinguishable from the solvent. Only those elements which differ in scattering length density from the solvent will effectively scatter neutrons. The density of the solvent can be changed readily through the use of deuterated water with minimal perturbation of the system. The range of neutron scattering length density available by this technique encompasses the range of densities for common biological molecules including DNA, RNA, proteins and lipids. This technique has been successfully exploited to examine the structures of molecules of heterogenous composition such as nucleosomes, ribosomes, viruses and protein-lipid complexes.

$\mathrm{X}$-rays are scattered by electrons and as a result electron-dense molecules such as DNA scatter much more intensely than less electron-dense molecules such as protein. Therefore, in the case of molecules of heterogeneous composition, scattering from the electron-dense regions predominates. While contrast matching is possible with $\mathrm{x}$-rays through the use of solutions containing sucrose or glycerol, in practice this can only be used to contrast match materials of low density such as proteins. Dense materials such as nucleic acids cannot be contrast matched. Additionally, very high concentrations of sucrose or glycerol must be used to achieve contrast matching and care must be taken to ensure that the structure of the molecule is not perturbed by the contrasting material.

The main advantage of $x$-ray scattering lies with the availability of synchrotron radiation sources which can provide intense fluxes of $\mathrm{x}$-rays compared to neutron fluxes from reactors or spallation sources. These high fluxes allow the collection of high quality data in a much shorter period of time. Synchrotron $x$-rays can be used to quickly screen different conditions and samples. Once an interesting structure has been identified, neutrons can be used to further probe and refine the structure. In this way, $\mathrm{x}$-ray and synchrotron radiation can be used in a complementary, time- and cost-effective manner to determine the structure of biological molecules and the effects of various environmental factors on their structures. 
Small angle solution scattering has played an important role in the elucidation of chromatin structure. Chromatin is composed of DNA and protein in well localized units, and is an ideal system to be examined by contrast matching techniques. Low resolution neutron scattering experiments determined the solution structure of the nucleosome core particle to be a flat disc $11.0 \mathrm{~nm}$ in diameter by 5.5 to $6.0 \mathrm{~nm}$ thick with 1.7 turns of DNA of pitch $3.0 \mathrm{~nm}$ coiled on the outside of the histone octamer. The x-ray crystal structures have been determined and confirm the low resolution solution structure and, in addition, give details of the histone DNA interactions and the path of the DNA. Of particular interest is the finding that DNA is not uniformly coiled but follows a path of coiled DNA segments followed by DNA bends. Such a DNA path has implications for the positioning of nucleosome on the underlying DNA sequence.

The structure of a simple gene consists of a transcription promotor, protein coding DNA sequences, and histone proteins. The histone proteins package the gene into higher order chromatin complexes. The transcription machinery recognizes protein factors bound to the promotor elements and copies the DNA coding sequences into RNA which serves as the template for protein synthesis. During human development particular patterns of gene expression are established and, as a result, differentiated cells do not express all the available genes. Cancer is an extreme example of the disruption of the normal patterns of gene expression. A growing body of evidence suggests that chromatin structure is directly involved in determining which genes are expressed in the cell.

Although histones are among the most highly conserved protein sequences in nature, families of histone subtypes provide for considerable variation in the packaging of DNA. There are 6 subtypes of human $\mathrm{H} 1,4$ subtypes of $\mathrm{H} 2 \mathrm{~A} ; 3$ subtypes of $\mathrm{H} 2 \mathrm{~B}, 3$ subtypes of $\mathrm{H} 3$ and 1 (possibly 2) $\mathrm{H} 4$ subtype(s). Many of these subtypes are cell-cycle dependent and package the genome during the S-phase of the cell cycle. Other subtypes e.g. H1, H2A.X, H2A.Z, H3.3 are cell-cycle independent and are thought to be required for the nucleosome packaging of active and potentially active genes of a particular cell type. These subtypes comprise only a few percent of the total histone in somatic cells.

Human sperm provide an extreme example of the different states of packaging of the human genome. In sperm $85 \%$ of the genome is packaged by protamines and is highly condensed. The remaining $15 \%$ of the genome is packaged by histones. I have demonstrated that this packaging is sequence specific and that histones $\mathrm{H} 2 \mathrm{~A}$ and $\mathrm{H} 2 \mathrm{~B}$ are comprised entirely of the subtypes found associated with active genes in somatic cells. Based on these results, I have hypothesized that the unusual genome organization in human sperm contributes to specific patterns of gene expression. 
I proposed a detailed SAXS investigation of nucleosomes containing the minor histone variants. H2A.X, H2A.Z, H2B.0, and $\mathrm{H} 3.3$ were used in reconstitutions with 195bp nucleosome positioning DNA. These particles were compared to control particles containing H2A.1, H2B.1, H3.1, and H4. Data analysis included determination of Rg from low $\mathrm{Q}$ data, and $\mathrm{P}(\mathrm{r})$ analysis of the entire scatter curves. Model fitting was used in structure refinements. One of the unusual properties of chromatin isolated from human sperm is the quantitative absence of linker histone H1. Instead of H1, operative chromatin from human sperm is stabilized by three high-molecular-weight proteins. I proposed to determine what these proteins were and investigate the structural properties of this complex.

\section{Importance to LANL's Science and Technology Base and National R\&D Needs}

This project supports both the Neutron Laboratory and Great Science tactical goals at Los Alamos. As stated above, neutrons provide a valuable research tool for large biological complexes. The successful utilization of the Los Alamos Neutron Science Laboratory (LANSCE) in addressing fundamental biological problems will result in increased support within the scientific community for the user facility. This is particularly important if LANL is to successfully compete for the establishment of a 5-10 megawatt spallation neutron source at Los Alamos. The establishment of a successful structural biology capability will support the Laboratory's core competency in bioscience and biotechnology, as well as enhance the Laboratory's ability to attract funding for fundamental research in this area. This project includes collaborations with investigators at Stanford University, University of California at Davis, and NIST.

\section{Scientific Approach and Accomplishments}

One of the aims of this project was to determine the proteins responsible for stabilizing operative chromatin. This chromatin is stabilized by microtubule proteins tau and tubulin. Using Western analysis, mononucleosomes were shown to contain tau protein. The mononucleosomes were then linked into higher order complexes by $\alpha$ and $\beta$ tubulin. Another goal of this project was the structural characterization of operative chromatin nucleosomes. Using solution scattering, nucleosomes containing the minor variants were shown to be structurally distinct from major variant containing nucleosomes. The nucleosomes containing minor variants had $\mathrm{D}_{\max }$ approximately $25 \mathrm{~A}$ larger than 
control particles. The unusual structure and stabilization of operative chromatin by microtubule proteins provides a possible mechanism for direct interaction of transcription machinery with specific chromatin domains. 